\title{
Predictors of post-operative response to treatment: a double blind placebo controlled study in chronic rhinosinusitis patients*
}

\author{
F.A. Ebbens ${ }^{1 \S}$, S. Toppila-Salmi ${ }^{2,3,4 \S}$, E.J.J. de Groot ${ }^{1}$, J. Renkonen ${ }^{3}$, \\ R. Renkonen ${ }^{3}$, C.M. van Drunen ${ }^{1}$, M.G.W. Dijkgraaf ${ }^{5}$, W.J. Fokkens ${ }^{1}$
}

1 Department of Otorhinolaryngology, Academic Medical Center, Amsterdam, the Netherlands

2 Department of Eye, Ear and Oral Diseases, Tampere University Hospital and University of Tampere, Tampere, Finland

3 Transplantation Laboratory \& Infection Biology Research Program, Haartman Institute, University of Helsinki and HUSLAB, Helsinki University Central Hospital, Helsinki, Finland

4 Department of Otorhinolaryngology, Helsinki University Central Hospital, Hyvinkaa Hospital, Hyvinkaa, Finland. Clinical Epidemiology, Biostatistics and Bioinformatics, Academic Medical Center,

5 Department of Clinical Epidemiology, Biostatistics and Bioinformatics, Academic Medical Center, Amsterdam, the Netherlands

SUMMARY

Background: In the majority of CRS patients suffering from primary or recurrent CRS, topical glucocorticoids are highly effective. A subset of CRS patients, however, does not respond to (topical) glucocorticoids and requires surgical intervention. Although surgery is highly effective in those individuals, recurrence of disease is observed in some. In this study we describe our search for one or more predictors predicting the response to surgery in combination with peri-operative oral glucocorticoids in CRS patients.

Methods: Thirty-five inferior turbinate specimens were randomly selected from a larger group of CRS patients requiring FESS for persistent disease that either responded favorably or demonstrated recurrent disease. Tissue biopsies were taken at the time of surgery and compared for inflammatory markers, endothelial cell markers, and various leukocyte subsets using immunohistochemistry.

Results: Compared to non-responders, the baseline level of lamina propria activated eosinophils is significantly increased in CRS patients responding to surgery in combination with peri-operative oral glucocorticoids treated or not treated post-operatively with topical glucocorticoids. No significant differences were observed for all other studied parameters. Post-operative treatment with FPANS $100 \mu \mathrm{g}$ q.i.d. was significantly associated with response to treatment. A trend towards association was observed for increased numbers of eosinophils at baseline.

Conclusion: Our data suggest that CRS patients with higher levels of eosinophils are less likely to suffer from post-operative recurrent sinonasal disease when treated post-operatively with FPANS $100 \mu$ g q.i.d.

Key words: chronic rhinosinusitus, nasal polyps, topical glucocorticoids

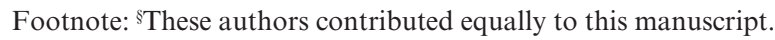




\section{INTRODUCTION}

Chronic rhinosinusitis (CRS) is an inflammatory disease of the nose and paranasal sinuses that is present for at least 12 weeks without complete resolution. It is characterized by the presence of distinctive symptoms (e.g. nasal blockage, nasal discharge, facial pain and/or reduced sense of smell) and either endoscopic signs or computed tomography (CT)-changes characteristic of the disease ${ }^{(1)}$. It contributes to a significant amount of health care expenditure as a result of direct costs arising from physician visits and medical therapies, as well as indirect costs related to loss of productivity and absence from work ${ }^{(2)}$.

CRS is a heterogeneous group of diseases with potentially different underlying etiologies and pathomechanisms. So far, CRS is clinically divided in CRS with nasal polyposis and CRS without nasal polyposis based on the presence of nasal polyps upon nasal endoscopy.

In the majority of CRS patients suffering from primary or recurrent CRS with or without nasal polyposis, topical glucocorticoids are highly effective ${ }^{(3-8)}$. A subset of CRS patients, however, does not respond to (topical) glucocorticoid therapy, even when therapy is prolonged, and is at risk for escalation of disease severity ${ }^{(9)}$. Ideally, non-responding patients are identified prior to the start of topical glucocorticoid therapy so that alternative treatment regimens can be considered from the onset.

Tissue specimens obtained from patients suffering from CRS with and without nasal polyposis are characterized by an influx of inflammatory cells, including (activated) eosinophils, neutrophils, and macrophages. Recruitment of some of these cells has been shown to involve inflammatory cell L-selectins and endothelial cell L-selectin counterreceptors (i.e. L-selectin ligands) ${ }^{(10,11)}$. In this study we describe our search for a predictor of response to surgery in combination with peri-operative oral glucocorticoids in a mixed group of CRS patients with and without nasal polyposis.

\section{METHODS}

\section{Participants}

This double-blind, placebo-controlled trial investigated the effectiveness of intranasal fluticasone propionate aqueous nasal spray (FPANS, either $100 \mu$ g q.i.d. or $200 \mu$ g q.i.d.) or placebo aqueous nasal spray (PANS) q.i.d. following functional endoscopic sinus surgery (FESS) in combination with peri-operative oral glucocorticoids, used for 12 months in adult patients with CRS with or without nasal polyposis. This study was prepared, conducted, and reported in compliance with the EU Note for Guidance on Good Clinical Practice as laid down in the Dutch Medicines Law (Wet op de Geneesmiddelenvoorziening, art. 55) and was approved by the medical ethical committee of the Erasmus Medical Center (Rotterdam, the Netherlands). Recruitment of patients took place from October 1994 to December 1997 at the department of Otorhinolaryngology of the Erasmus Medical Center,
Rotterdam, the Netherlands, a tertiary Otorhinolaryngology clinic. Prior to enrollment, each patient was required to read and sign an informed consent form. All patients aged 18 years or older with clinical signs and symptoms related to CRS (e.g. nasal blockage, nasal discharge, and/or facial pain) requiring surgery, endoscopic signs and sinus computed tomography (CT)-scan findings consistent with CRS with nasal polyposis or CRS without nasal polyposis (i.e. a sinus CT-scan score of at least 2 (e.g. obstruction of at least one ostiomeatal complex) according to the Lund and Mackay scoring system $\left.{ }^{(12)}\right)$ were eligible to enroll provided that none of the exclusion criteria was present (Table 1). Nasal endoscopy was performed using a $30^{\circ}$ Storz nasendoscope (Karl Storz GmbH, Tuttlingen, Germany) after local anesthesia and decongestion with novesin and xylomethazoline. All patient-related study data were recorded in Case Report Forms and internally monitored for accuracy and completeness.

\section{Intervention}

Each patient underwent a FESS procedure according to the extent of his or her disease ${ }^{(13)}$. In all cases, the ostiomeatal complex was opened. If necessary, the procedure included opening to the ethmoidal, frontal and/or sphenoidal sinuses. In case a severe septal deviation was present, septoplasty was performed. All patients received a dexamethasone booster course starting with $1 \mathrm{mg}$ q.i.d. at the day of surgery and tapering off with $0.5 \mathrm{mg} /$ day every second day. The use of systemic steroids was not allowed in the remainder of this study. Following FESS (visit 2) and removal of the ethmoidal packing (visit 3, 1 week after surgery), patients applied either FPANS $100 \mu \mathrm{g}$ (i.e. 2 actuations) q.i.d., FPANS $200 \mu$ g (i.e. 2 actuations) q.i.d., or PANS ( 2 actuations) q.i.d. into each nostril. Randomized allocation to one of the three treatment groups took place after removal of the ethmoidal packing on visit 3 (one week after surgery). Follow-up visits were scheduled at 2, 4, 8, 12, 18, $26,32,39,45$, and 52 weeks after surgery. Upon randomization and at each clinic visit, the hospital pharmacist provided each participating patient with trial medication. Trial medication was instructed to be stored in a secure area between $2{ }^{\circ} \mathrm{C}$ and $25^{\circ} \mathrm{C}$, free of environmental extremes. All groups continued current treatment regimens for conditions other than CRS with or without nasal polyposis. Dosages of these treatment regimens were kept constant throughout the period of participation in this study whenever possible and patients were instructed to record and report any changes. The use of systemic steroids was not allowed except for a dexamethasone booster course starting with $1 \mathrm{mg}$ q.i.d. at the day of surgery and tapering off with $0.5 \mathrm{mg} /$ day every second day. Antibiotics (e.g. amoxicillin/clavulanic acid 500/125 mg t.i.d.) were allowed upon exacerbation of disease when necessary. In case of severe symptoms, those patients suffering from concurrent allergic rhinitis were allowed to use terfenadine $60 \mathrm{mg}$ as rescue medication should their symptoms of allergy become troublesome (maximum 2 tablets a day). Upon randomization, the use of the nasal spray pump was explained to each participating patient. Patients were instructed to actuate the spray twice into 
each nostril in the morning before breakfast and twice into each nostril after breakfast. Patients were instructed to actuate the spray into each nostril in a similar way and dose before and after dinner. Compliance was checked by looking at the amount of residual spray solution returned to the pharmacist at each clinic visit.

\section{Objectives and outcome}

Clinical outcomes of this study were recently published in Clinical and Experimental Allergy ${ }^{(14)}$. The purpose of our present study was to obtain one or more baseline immunohistochemical parameters (thus prior to the start of treatment with FPANS or PANS) that could predict the recurrence or persistence of CRS with or without nasal polyposis. Nonresponders were identified in case of recurrent or persistent disease, which was defined as a progressive regrowth of nasal polyps, recurrent complaints of CRS combined with signs of CRS upon endoscopy and abnormalities on a sinus CT-scan, or persistent complaints of CRS with or without nasal polyposis for at least two months after FESS. Individuals withdrawn from the study due to the occurrence of an adverse event or due to poor compliance were not considered nonresponders.

\section{Randomization}

Following FESS, patients were randomly allocated at visit 3 to one of the three treatment groups (ratio 1:1:1) using a computer generated randomization schedule provided by the Department of Biostatistics, Erasmus University Medical Center, Rotterdam, the Netherlands. Patient numbers were sequentially assigned in time. Numbered aqueous nasal spray bottles were dispensed by an independent pharmacist to each patient upon randomization and at each clinic visit. All study personnel and participants were blinded to experimental conditions for the duration of the study. Randomization codes were revealed to the researchers only when recruitment and data collection were complete.

\section{Statistical analysis}

Analysis was based on thirty-five patients that had been randomly assigned to one of three treatment arms. One-way ANOVA and chi-square analyses were performed to check for between-group demographic differences between the three treatment groups. Two-sided T-tests and Mann-Whitney-U non-parametric tests were performed to check for betweengroup differences in clinical and cellular characteristics at baseline (i.e. at the time of surgery) between non-responders and responders. Spearman rank-order correlation was used to assess bivariate association. Immunohistochemical data are represented as median (interquartile range). A binary logistic regression analysis and the van Elteren test were performed to study the association between cellular markers at baseline and successful response to treatment with surgery in combination with peri-operative oral glucocorticoids and with or without topical glucocorticoids. P values $<0.05$ were considered statistically significant. All statistical analyses were performed using SPSS 16.0 (Chicago, IL, USA).

\section{Tissue handling}

Samples of inferior turbinate obtained at the time of surgery were immediately snap-frozen in liquid nitrogen and stored at $-80^{\circ} \mathrm{C}$ until analysis.

\section{Immunohistochemical staining}

Six- $\mu \mathrm{m}$ thick serial sections of all snap-frozen specimens were cut on a Microm HM560 frigocut cryostat and transferred to APES (amino-phosphate-ethylsilane) coated slides (Starfrost, MarketLab, Caledonia, MI, USA), dried and stored at $-70^{\circ} \mathrm{C}$ until analysis. Immunohistochemical analysis of MBP, CD14, CD34 and functionally active L-selectin ligands was based on the alkaline phosphatase method described previously by our group ${ }^{(11)}$. For basogranulin, ECP/EDN, IL-5, CD15 and CD94, a sensitive protocol, based on the above mentioned alkaline phosphatase method, was used ${ }^{(11)}$. Antibodies used are summarized in Table 2.

\section{Light microscope evaluation}

All sections were examined with an Olympus BX51 light microscope by 2 independent observers blinded to the experimental conditions. The numbers of positively stained cells were counted in the epithelium (per $\mathrm{mm}$ ) and adjacent lamina propria (per $\mathrm{mm}^{2}$ ) at a final magnification of 200x. Results are expressed as the mean number of positive cells per $\mathrm{mm}$ or $\mathrm{mm}^{2}$.

\section{RESULTS}

Participants

Of the 162 patients included in this double-blind placebocontrolled multicenter trial, 59 patients completed the study without signs of recurrent disease (i.e. no signs of progressive regrowth of nasal polyps, recurrent complaints of CRS combined with signs of CRS upon endoscopy and sinus CT scan abnormalities, or persistent complaints of CRS with or without nasal polyposis for at least two months after FESS). Thirty-five patients were randomly selected from the 162 patients included in this trial, including both responders and non-responders (14 from the PANS treated group, 9 from the FPANS $100 \mu$ g q.i.d. treated group and 12 from the FPANS $200 \mu$ g q.i.d. treated group). Biopsies were obtained at the time of surgery (visit 2). No significant differences in demographic and clinical characteristics (a.o. the presence or absence of nasal polyps) were observed at baseline (i.e. at the time of surgery) between the three treatment groups (Table 3). When comparing demographic and clinical characteristics at baseline between non-responders and responders, similar results were obtained (data not shown). Prior surgery was shown not to influence outcome (data not shown).

\section{Cellular markers at baseline}

In the first part of our study, patients were divided into responders and non-responders based on the criteria described above. Endothelial expression levels of functionally active L-selectin ligands were analyzed using two anti-glycan antibodies (mAb MECA-79 and mAb HECA-452). In addition, the number of $\mathrm{CD} 34+$ endothelial cells was determined in all 
Table 1. Exclusion criteria.

Cystic fibrosis
Patients taking systemic glucocorticoids
Patients taking oral medications that are known to interfere with
nasal mucosa hyperplasia (e.g. cyclosporin)
Patient taking intranasal medications other than the trial
medication
Contraindications for the use of (topical) glucocorticoids
Nasal surgery (including FESS) in the 6 weeks prior to inclusion
Any serious or unstable concurrent disease
Any structural nasal abnormalities (other than CRS with or
without NP)
Aspirin intolerance
Females who are pregnant or lactating or are likely to become
pregnant during the study
Inability to follow the instructions within the protocol or known
inability to attend all clinical visits within the intervals stated

tissue specimens. Other markers of inflammation that were quantified included the numbers of IL-5+ cells, eosinophils, neutrophils, macrophages, NK-cells and basophils (Tables 4 and 5). Compared to non-responders, the number of lamina propria EG2+ eosinophils (i.e. activated eosinophils) is significantly increased in the responders group $(p=0.005)$. This increase in the number of EG2+ eosinophils is present in both CRS patients without nasal polyposis and CRS patients with nasal polyposis. In addition, a trend towards significance is observed for the number of lamina propria CD15+ neutrophils ( $p=0.066)$ in the responders group. The number of epithelial CD14+ macrophages, on the other hand, is significantly increased in the non-responders group $(\mathrm{p}=0.043)$. No differences were observed for all other studied parameters (Tables 4 and 5). Non-responders and responders did not differ in the percentage CD34+ vessels positive for mAb MECA-79 (2.92\% (IQ-range $0.73-9.91$ ) and $1.62 \%$ (IQ-range $0.30-5.77$ ), respectively) and $\mathrm{mAb}$ HECA-452 (0.33\% (IQ-range 0.00 -1.30 ) and $1.02 \%$ (IQ-range $0.33-1.48$ ), respectively). No correlation was observed between the percentage of CD34+ vessels positive for mAb MECA-79 and mAb HECA-452 and all studied inflammatory cells.

\section{Predictors of response}

The main goal of this study was to identify one or more predictors that could predict the outcome of surgery in combination with peri-operative oral glucocorticoids in CRS patients with or without nasal polyposis within our data pool. A binary logistic regression analysis and the van Elteren test were performed to identify one or more of these predictors. Since only 21 patients were included in the non-responders group and 14 in the responders group, only 2 predictors were allowed in our binary logistic regression analysis. Both treatment with topical glucocorticoids and the number of lamina propria EG2+ eosinophils were identified as possible predictors of response. Only treatment with topical glucocorticoids was significantly $(\mathrm{p}=0.048)$ associated with response in our model including treatment with topical glucocorticoids and the number of lamina propria EG2+ eosinophils. The impact of treatment with topical glucocorticoids was solely attributable to the FPANS $100 \mu$ g q.i.d. regimen compared to placebo (odds ratio
9.9; 95\% CI 1.1 - 88.8). Although significantly higher baseline levels of lamina propria EG2+ eosinophils were observed in the responders group (Figure 1), in our model this difference only showed a trend towards significance but did not reach significance yet $(p=0.091)$. Using the Van Elteren test, comparing baseline levels of lamina propria EG2+ eosinophils in non-responders and responders stratified by post-operative treatment with placebo, FPANS $100 \mu$ g q.i.d. or FPANS 200 $\mu \mathrm{g}$ q.i.d., higher baseline levels of lamina propria EG2+ eosinophils were however significantly associated with a positive response to surgery in combination with peri-operative oral glucocorticoids $(\mathrm{p}=0.00035)$.

\section{DISCUSSION}

Fluticasone propionate is a glucocorticoid with high topical activity and low systemic bioavailability. It is considered one of the most potent topical glucocorticoids currently available for the treatment of patients suffering from CRS with or without nasal polyposis. Various controlled trials have demonstrated that treatment with fluticasone propionate is well tolerated and effective in the majority of CRS patients with and without nasal polyposis ${ }^{(3-6,8)}$. Besides being effective in treating signs and symptoms of primary and recurrent CRS, the use of fluticasone propionate has been shown to effectively prevent post-surgical recurrence of disease ${ }^{(15)}$. Only two studies, one of which was recently published and describes the clinical results of this study ${ }^{(14)}$, were unable to demonstrate a positive effect of fluticasone propionate on outcome ${ }^{(14,16)}$.

It is generally accepted that topical glucocorticoids reduce the number of inflammatory cells in CRS mucosa, particularly the numbers of primed eosinophils. This may be achieved either directly via a reduction in cell viability and activation ${ }^{(17,18)}$ or indirectly via a reduction in the secretion of chemotactic cytokines ${ }^{(19-21)}$. The exact mechanisms underlying the antiinflammatory and immunoregulatory effects of topical glucocorticoids, however, remain to be fully explained.

Although most patients respond well, a substantial number of CRS patients with and without nasal polyposis is refractory to (topical) glucocorticoids and requires surgical intervention. Both increased disease severity and/or glucocorticoid resistance are likely involved. The exact cause of glucocorticoid resistance is unknown. Overexpression of glucocorticoid receptor $\beta$ and/or a downregulation of glucocorticoid receptor $\alpha$ are two of the mechanisms that have been proposed ${ }^{(22,23)}$. Other mechanisms include immunomodulation, cigarette smoking, genetic predisposition, viral infection, allergen exposure, the presence of microbial superantigens, and tissue neutrophilia (24). Ideally, those patients resistant to glucocorticoids are identified prior to starting topical glucocorticoid treatment. If identified early, those patients resistant to topical glucocorticoids can be treated alternatively with, for example, long-term macrolide antibiotics ${ }^{(25,26)}$. With this in mind, we aimed to identify a cellular marker predicting the response to surgery in combination with peri-operative oral glucocorticoids with or 
Table 2. Monoclonal antibodies.

\begin{tabular}{lcccc}
\hline \multicolumn{1}{c}{ Antibody } & Specificity & Cell type/ cytokine & Titer & Source \\
\hline$\alpha$-CD14 & CD14 & Macrophages & $1: 600$ & CLB, NL \\
$\alpha$-CD15 & CD15 & Neutrophils & $1: 50$ & Immunotech, France \\
$\alpha$-CD34 & CD34 & Endothelial cells & $1: 100$ & DAKO, Denmark \\
$\alpha$-CD94 & ND-cells & $1: 25$ & Beckman Coulter, NL \\
BB-1 & Basogranulin & Basophils & $1: 150$ & A.F. Walls, UK \\
BMK-13 & MBP & Eosinophils & $1: 100$ & CLB, Netherlands \\
EG2 & ECP/EDN & Activated & $1: 100$ & Sanbio, Netherlands \\
$\alpha$-IL-5 & eosinophils & $1: 100$ & Novartis,Switzerland \\
HECA-452 & IL-5 & L-selectin ligand & $1: 100$ & BD Biosciences, USA \\
& 2,3-sialylation \& 1,3- & & \\
fucosylation of lactosamine & & & BD Biosciences, USA \\
\hline
\end{tabular}

Table 3. Baseline characteristics of the study population $(n=35)$.

\begin{tabular}{|c|c|c|c|c|}
\hline & $\begin{array}{l}\text { Placebo } \\
(\mathrm{n}=14)\end{array}$ & $\begin{array}{c}\text { FPANS } 400 \mu \mathrm{g} / \text { day } \\
(\mathrm{n}=9)\end{array}$ & $\begin{array}{c}\text { FPANS } 800 \mu \mathrm{g} / \mathrm{day} \\
(\mathrm{n}=12)\end{array}$ & $\mathrm{p}$ value \\
\hline Age (y), mean (SD) & 48 (14) & $41(12)$ & $43(11)$ & 0.38 \\
\hline Male sex, n (\%) & $6(42.9)$ & $8(88.9)$ & $7(58.3)$ & 0.09 \\
\hline Asthma, n (\%) & $3(21.4)$ & $4(44.4)$ & $2(16.7)$ & 0.41 \\
\hline Atopy, $\mathrm{n}(\%)$ & $5(35.7)$ & $3(33.3)$ & $5(41.7)$ & 0.92 \\
\hline Previous sinus surgery, $\mathrm{n}(\%)$ & $8(57.1)$ & $4(44.4)$ & $6(50.0)$ & 0.83 \\
\hline Smoking habits & & & & 0.48 \\
\hline Current smoker, n (\%) & $3(21.4)$ & $2(22.2)$ & $4(33.3)$ & \\
\hline Ex-smoker, n (\%) & $1(7.1)$ & $0(0)$ & $2(16.7)$ & \\
\hline Non-smoker, n (\%) & $10(71.4)$ & $7(77.8)$ & $5(41.7)$ & \\
\hline CT scan score, mean (SD) & $12.9(5.7)$ & $12.7(6.0)$ & $10.9(3.8)$ & 0.61 \\
\hline Total VAS score, mean (SD) & $227.6(106.7)$ & $199.8(96.6)$ & $261.8(93.7)$ & 0.37 \\
\hline Non-responders, $\mathrm{n}(\%)$ & $9(64.3)$ & $3(33.3)$ & $9(75.0)$ & 0.14 \\
\hline CRS with NP, n (\%) & $8(57.1)$ & $5(55.6)$ & $5(41.7)$ & 0.83 \\
\hline
\end{tabular}

without post-operative treatment with fluticasone propionate aqueous nasal spray in a mixed group of CRS patients with and without nasal polyposis.

Inferior turbinates are easy accessible and present in all CRS patients with and without nasal polyposis. We chose to investigate inferior turbinate biopsies, as middle turbinate specimens can be absent in those CRS patients suffering from recalcitrant disease. Levels of various inflammatory mediators, including IL-5, were recently shown to be equally present in inferior turbinate specimens and nasal polyp tissue specimens ${ }^{(27)}$. Thus, inferior turbinate tissue specimens seem to be ideal for those doctors wanting to predict response to treatment in nasal biopsies.

Baseline (i.e. at the time of surgery) inferior turbinate specimens of responders are characterized by high levels eosinophils, NK-cells, neutrophils, and an increase in the number of IL-5+ cells. Baseline inferior turbinate tissue specimens of non-responders, on the other hand, are characterized by high levels of basophils, NK-cells and macrophages (Table 4). Tissue specimens from both groups are characterized by high levels of CD34+ endothelial cells. Only a small percentage of these CD34+ endothelial cells do express functionally active L-selectin ligands (as detected by both mAb HECA-452 and mAb MECA-79). Although we have previously demonstrated that, in CRS patients without nasal polyposis, the extent of tissue eosinophilia correlates with the percentage of CD34+ endothelial cells expressing functionally active L-selectin ligands ${ }^{(10)}$, no correlation was observed between the percentage of CD34+ endothelial cells expressing functionally active L-selectin ligands and any of the studied inflammatory cells in this study. Although a lack of power cannot be ruled out, results are in line with data obtained in a second study from our group investigating nasal polyps from CRS patients with nasal polyposis ${ }^{(11)}$.

Both treatment with fluticasone propionate and the number of lamina propria EG2+ eosinophils were identified as possible predictors of response to surgery in combination with peri-operative oral glucocorticoids. Only treatment with fluticasone proprionate was significantly $(\mathrm{p}=0.048)$ associated with response in our model including treatment with fluticasone proprionate and the number of lamina propria EG2+ eosinophils as variables. The impact of treatment with fluticasone proprionate was solely attributable to the FPANS $100 \mu$ g q.i.d. regimen compared to placebo. These results are in line with results published by Rowe-Jones et al. who observed that CRS patients with or without nasal polyposis performed significantly better on all outcome measures when treated with fluticasone propionate $200 \mu \mathrm{g}$ b.i.d. (a similar daily dose as our study) for 4 years following FESS in a randomized, stratified, 
Table 4. Number of positive cells per mm epithelium in inferior turbinate specimens at baseline (median - IQ range).

\begin{tabular}{lcc}
\hline & Responders & Non-Responders \\
\hline Eosinophils (MBP+) & $1.00(0.00-2.57)$ & $0.05(0.00-0.49)$ \\
Activated eosinophils (ECP/ EDN+) & $0.00(0.00-0.00)$ & $0.00(0.00-0.00)$ \\
Basophils ( basogranulin+) & $0.72(0.00-2.50)$ & $2.50(0.00-5.90)$ \\
NK-cells (CD94+) & $3.53(1.54-7.26)$ & $4.46(0.78-11.27)$ \\
Neutrophils (CD15+) & $2.67(0.34-7.81)$ & $1.30(0.55-3.91)$ \\
Macrophages (CD14+) & $0.79(0.00-2.42) \dagger$ & $3.00(0.70-8.57) \dagger$ \\
IL-5 & $2.73(0.59-5.54)$ & $0.88(0.00-2.94)$ \\
\hline
\end{tabular}

$\uparrow \mathrm{p}<0.05$ and $\$ \mathrm{p}<0.01$

Table 5. Number of positive cells per $\mathrm{mm}^{2}$ lamina propria in inferior turbinate specimens at baseline (median - IQ range).

\begin{tabular}{|c|c|c|}
\hline & Responders & Non-Responders \\
\hline Eosinophils (MBP+) & $11.38(0.96-24.55)$ & $1.74(0.33-11.94)$ \\
\hline Activated eosinophils (ECP/ EDN+) & $0.64(0.11-1.39) \ddagger$ & $0.00(0.00-0.00) \$$ \\
\hline Basophils ( basogranulin + ) & $9.40(1.08-15.38)$ & $9.06(0.00-17.90)$ \\
\hline NK-cells (CD94+) & $14.29(7.40-27.75)$ & $11.18(2.87-16.20)$ \\
\hline Neutrophils (CD15+) & $14.38(5.85-24.33)$ & $5.96(1.51-41.05)$ \\
\hline Macrophages (CD14+) & $10.47(1.09-25.80)$ & $16.47(4.14-25.78)$ \\
\hline IL-5 & $16.47(2.66-28.94)$ & $11.23(3.51-23.50)$ \\
\hline
\end{tabular}

$\uparrow \mathrm{p}<0.05$ and $\$ \mathrm{p}<0.01$

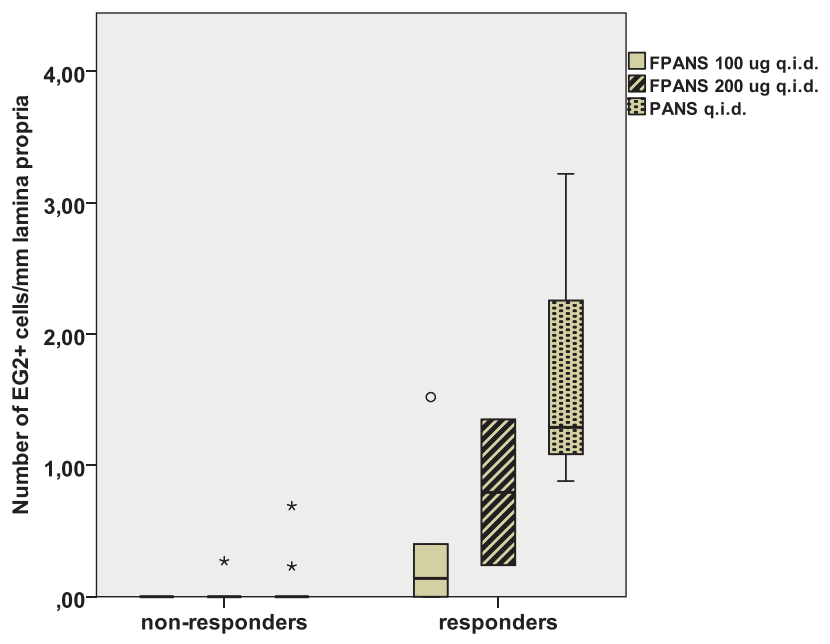

Figure 1. Predictors of response to treatment.

prospective, double-blind, placebo controlled study ${ }^{(15)}$, but do not confirm the overall results of our clinical data (in which responders and non-responders were grouped together) ${ }^{(14)}$. Our current division in responders and non-responders possibly explains the observed results since the majority of patients presented in our clinical study belonged to the non-responders group. A selection bias, however, cannot be excluded. Those patients treated with FPANS $200 \mu$ g q.i.d. and PANS q.i.d. post-operatively are more likely to suffer from recurrent disease, results in line with our clinical data ${ }^{(14)}$.
Recently, increased numbers of eosinophils were shown to be characteristic of both patients suffering from CRS with nasal polyposis and patients suffering from CRS without nasal polyposis. Levels, however, were shown to vary between individuals and within biopsies (28). In this study, we show that those CRS patients with increased numbers of lamina propria EG2+ eosinophils are more likely to respond to surgery, especially when treated post-operatively with FPANS $100 \mu$ g q.i.d., than those CRS patients with low numbers of lamina propria EG2+ eosinophils. In our model including treatment with fluticasone proprionate and the number of lamina propria EG2+ eosinophils as variables this difference only showed a trend towards significance but did not reach significance yet $(\mathrm{p}=0.091)$. However, using the Van Elteren test, comparing baseline levels of lamina propria EG2+ eosinophils in non-responders and responders stratified by postoperative treatment with placebo, FPANS $100 \mu$ g q.i.d. or FPANS $200 \mu$ g q.i.d., higher baseline levels of lamina propria EG2+ eosinophils were significantly associated with a positive response to surgery in combination with peri-operative oral glucocorticoids $(p=0.00035)$. These results are in line with data observed in symptomatic asthmatic patients, in whom low sputum eosinophils were shown to predict the lack of response to topical beclomethasone ${ }^{(29)}$. Therefore, additional research in CRS patients with and without nasal polyposis studying the role of EG2+ eosinophils and response to FESS in combination with peri-operative oral glucocorticoids and post-operative fluticasone propionate treatment is warranted. 


\section{REFERENCES}

1. Fokkens W, Lund V, Mullol J. European position paper on rhinosinusitis and nasal polyps 2007. Rhinol Suppl. 2007; 1-136.

2. Lanza DC, Kennedy DW. Adult rhinosinusitis defined. Otolaryngol Head Neck Surg. 1997; 117(3 Pt 2): S1-S7.

3. Holmberg K, Juliusson S, Balder B, Smith DL, Richards DH, Karlsson G. Fluticasone propionate aqueous nasal spray in the treatment of nasal polyposis. Ann Allergy Asthma Immunol. 1997; 78: 270-6.

4. Lund VJ, Flood J, Sykes AP, Richards DH. Effect of fluticasone in severe polyposis. Arch Otolaryngol Head Neck Surg. 1998; 124: 513-518.

5. Keith P, Nieminen J, Hollingworth K, Dolovich J. Efficacy and tolerability of fluticasone propionate nasal drops 400 microgram once daily compared with placebo for the treatment of bilateral polyposis in adults. Clin Exp Allergy. 2000; 30: 1460-1468.

6. Penttila M, Poulsen P, Hollingworth K, Holmstrom M. Doserelated efficacy and tolerability of fluticasone propionate nasal drops 400 microg once daily and twice daily in the treatment of bilateral nasal polyposis: a placebo-controlled randomized study in adult patients. Clin Exp Allergy. 2000; 30: 94-102.

7. Lund VJ, Black JH, Szabo LZ, Schrewelius C, Akerlund A. Efficacy and tolerability of budesonide aqueous nasal spray in chronic rhinosinusitis patients. Rhinology. 2004; 42: 57-62.

8. Aukema AA, Mulder PG, Fokkens WJ. Treatment of nasal polyposis and chronic rhinosinusitis with fluticasone propionate nasal drops reduces need for sinus surgery. J Allergy Clin Immunol. 2005; 115: 1017-1023.

9. van Camp C, Clement PA. Results of oral steroid treatment in nasal polyposis. Rhinology. 1994; 32: 5-9.

10. Toppila-Salmi SK, Myller JP, Torkkeli TV, et al. Endothelial L-selectin ligands in sinus mucosa during chronic maxillary rhinosinusitis. Am J Respir Crit Care Med. 2005; 171: 1350-1357.

11. Ebbens FA, Toppila-Salmi SK, Renkonen JA et al. Endothelial L-selectin ligand expression in nasal polyps. Allergy. 2010; 65: 95-102.

12. Lund VJ, Mackay IS. Staging in rhinosinusitus. Rhinology. 1993; 31: 183-184.

13. Stammberger H, Posawetz W. Functional endoscopic sinus surgery. Concept, indications and results of the Messerklinger technique. Eur Arch Otorhinolaryngol. 1990; 247: 63-76.

14. Dijkstra MD, Ebbens FA, Poublon RM, Fokkens WJ. Fluticasone propionate aqueous nasal spray does not influence the recurrence rate of chronic rhinosinusitis and nasal polyps 1 year after functional endoscopic sinus surgery. Clin Exp Allergy. 2004; 34: 1395 1400 .

15. Rowe-Jones JM, Medcalf M, Durham SR, Richards DH, Mackay IS. Functional endoscopic sinus surgery: 5 year follow up and results of a prospective, randomised, stratified, double-blind, placebo controlled study of postoperative fluticasone propionate aqueous nasal spray. Rhinology. 2005; 43: 2-10.

16. Parikh A, Scadding GK, Darby Y, Baker RC. Topical corticosteroids in chronic rhinosinusitis: a randomized, double-blind, placebo-controlled trial using fluticasone propionate aqueous nasal spray. Rhinology. 2001; 39: 75-79.

17. Mullol J, Xaubet A, Lopez E, Roca-Ferrer J, Picado C. Comparative study of the effects of different glucocorticosteroids on eosinophil survival primed by cultured epithelial cell supernatants obtained from nasal mucosa and nasal polyps. Thorax 1995; 50: $270-274$.
18. Mullol J, Lopez E, Roca-Ferrer J, et al. Effects of topical antiinflammatory drugs on eosinophil survival primed by epithelial cells. Additive effect of glucocorticoids and nedocromil sodium. Clin Exp Allergy 1997; 27: 1432-1441.

19. Roca-Ferrer J, Mullol J, Lopez E, et al. Effect of topical antiinflammatory drugs on epithelial cell-induced eosinophil survival and GM-CSF secretion. Eur Respir J. 1997; 10: 1489-1495.

20. Mullol J, Roca-Ferrer J, Xaubet A, Raserra J, Picado C. Inhibition of GM-CSF secretion by topical corticosteroids and nedocromil sodium. A comparison study using nasal polyp epithelial cells. Respir Med. 2000; 94: 428-431.

21. Xaubet A, Mullol J, Roca-Ferrer J, et al. Effect of budesonide and nedocromil sodium on IL-6 and IL-8 release from human nasal mucosa and polyp epithelial cells. Respir Med. 2001; 95: 408-414.

22. Hamilos DL, Leung DY, Muro S, et al. GRbeta expression in nasal polyp inflammatory cells and its relationship to the antiinflammatory effects of intranasal fluticasone. J Allergy Clin Immunol. 2001; 108: 59-68.

23. Pujols L, Mullol J, Benitez P, et al. Expression of the glucocorticoid receptor alpha and beta isoforms in human nasal mucosa and polyp epithelial cells. Respir Med. 2003; 97: 90-6.

24. Leung DY, Bloom JW. Update on glucocorticoid action and resistance. J Allergy Clin Immunol. 2003; 111: 3-22.

25. Ragab SM, Lund VJ, Scadding G. Evaluation of the medical and surgical treatment of chronic rhinosinusitis: a prospective, randomised, controlled trial. Laryngoscope. 2004; 114: 923-30.

26. Wallwork B, Coman W, kay-Sim A, Greiff L, Cervin A. A double-blind, randomized, placebo-controlled trial of macrolide in the treatment of chronic rhinosinusitis. Laryngoscope. 2006; 116: 189-193.

27. Danielsen A, Tynning T, Brokstad KA, Olofsson J, Davidsson A. Interleukin 5, IL6, IL12, IFN-gamma, RANTES and Fractalkine in human nasal polyps, turbinate mucosa and serum. Eur Arch Otorhinolaryngol 2006; 263: 282-9.

28. Ponikau JU, Sherris DA, Kern EB, et al. The diagnosis and incidence of allergic fungal sinusitis. Mayo Clin Proc. 1999; 74: 877-84.

29. Bacci E, Cianchetti S, Bartoli M, et al. Low sputum eosinophils predict the lack of response to beclomethasone in symptomatic asthmatic patients. Chest. 2006; 129: 565-72.

Fenna A. Ebbens, MD, PhD

Department of Otorhinolaryngology

Academic Medical Center

Meibergdreef 9

1105 AZ Amsterdam

The Netherlands

Tel: +31-20-566 3789

Fax: +31-20-566 9573

E-mail: f.a.ebbens@amc.uva.nl 